\title{
AMO EXPRESS: A Command and Control Experiment for Crew Autonomy
}

\author{
Howard K Stetson \\ Teledyne Brown Engineering \\ Huntsville, Al 35812 \\ Howard.K.Stetson@nasa.gov \\ Randy Cornelius \\ Teledyne Brown Engineering \\ Huntsville, Al 35812 \\ James.r.cornelius@nasa.gov
Lui Wang
NASA Johnson Space Center
Houston TX 77058
Lui.wang-1@nasa.gov

\author{
Jeremy Frank \\ NASA Ames Research Center. \\ Jeremy.d.Frank@nasa.gov \\ Angie Haddock \\ NASA Marshall Space Flight Center \\ Huntsville, Al 35812 \\ Angie.Haddock@nasa.gov \\ Larry Garner \\ Tietronix \\ Houston TX 77058 \\ Larry.w.garner@nasa.gov
}

NASA is investigating a range of future human spaceflight missions, including both Mars-distance and Near Earth Object (NEO) targets. Of significant importance for these missions is the balance between crew autonomy and vehicle automation. As distance from Earth results in increasing communication delays, future crews need both the capability and authority to independently make decisions. However, small crews cannot take on all functions performed by ground today, and so vehicles must be more automated to reduce the crew workload for such missions.

NASA's Advanced Exploration Systems Program funded Autonomous Mission Operations (AMO) project conducted an autonomous command and control demonstration of intelligent procedures to automatically initialize a rack onboard the International Space Station (ISS) with power and thermal interfaces, and involving core and payload command and telemetry processing, without support from ground controllers. This autonomous operations capability is enabling in scenarios such as a crew medical emergency, and representative of other spacecraft autonomy challenges. The experiment was conducted using the Expedite the Processing of Experiments for Space Station (EXPRESS) rack 7, which was located in the Port 2 location within the U.S Laboratory onboard the International Space Station (ISS). Activation and deactivation of this facility is time consuming and operationally intensive, requiring coordination of three flight control positions, 47 nominal steps, 57 commands, 276 telemetry checks, and coordination of multiple ISS systems (both core and payload).

The autonomous operations concept includes a reduction of the amount of data a crew operator is required to verify during activation or de-activation, as well as integration of procedure execution status and relevant data in a single integrated display. During execution, the auto-procedures provide a step-by-step messaging paradigm and a high level status upon termination. This messaging and high level status is the only data generated for operator display. To enhance situational awareness of the operator, the Web-based Procedure Display (WebPD) provides a novel approach to the issues of procedure display and execution tracking. For this demonstration, the 
procedure was initiated and monitored from the ground. As the Timeliner sequences executed, their high level execution status was transmitted to ground, for WebPD consumption.

The AMO EXPRESS experiment demonstrated activation and de-activation of EXPRESS rack 7, providing the capability of future single button activations and deactivations of facility class racks. The experiment achieved numerous technical and operations 'firsts' for the ISS, including:

- Integration of core and payload automation onboard ISS (Payload bundles communicating to core and payload avionics).

- Embedding of vehicle command and telemetry into an auto-procedure.

- Integration of nominal and off-nominal operations (embedded fault detection and recovery) in single procedure.

- Combined three console position procedures into two "Single Button" autonomous operations.

- Combined core and payload operations in a single autonomous procedure.

- Autonomous command response to a detected failure (two configuration files did not load; this was detected and file contents loaded autonomously). 